\title{
COGNITIVE DEVELOPMENT
}

\section{Reorientation in a small-scale environment by 3-, 4-, and 5-year-old children}

\author{
S. Gouteux ${ }^{\mathrm{a}}$, J. Vauclair ${ }^{\mathrm{b}}$, C. Thinus-Blanc ${ }^{\mathrm{a}, *}$ \\ ${ }^{a}$ Centre for Research in Cognitive Neuroscience, National Center for Scientific Research, \\ 31, Chemin Joseph Aiguier, 13402 Marseille Cedex 20, France \\ ${ }^{\mathrm{b}}$ Centre for Research in Psychology of Cognition, Language and Emotion, \\ Université de Provence, Aix-en-Provence, France
}

Received 1 May 2000; received in revised form 1 January 2001; accepted 1 May 2001

\begin{abstract}
Geometric features of surfaces and local information are constitutive elements of spatial representations. A number of studies in animals (rats) and human children (24 months old) have shown that in a rectangular environment with a reward hidden in one of the corners, geometric properties predominate over local cues for search strategies. In contrast, monkeys and human adults are able to take into account both types of information (geometric and local) to reorient. So far, all of the experiments have been conducted in the locomotor space involving a navigational task. In the present study, we examined whether similar search patterns are found using a tabletop model of a rectangular room. Three groups of children (3-, 4-, and 5-year-olds) and one group of adults were tested. Results show that geometric encoding appears only at 4 years of age, that is later than in the locomotor space. The joint use of geometry and local cues emerges at 5 years of age. These data show that similar types of processing are implemented in both manipulatory and locomotor space but not at the same time. The difference between locomotor and manipulatory tasks suggests that being immersed in the environment makes this separated processing easier than being confronted by a task for which the object is exterior to the participant. (C) 2001 Elsevier Science Inc. All rights reserved.
\end{abstract}

Keywords: Geometric encoding; Spatial cognition; Development

* Corresponding author. Fax: +33-91-77-49-69.

E-mail address: thinus@lnf.cnrs-mrs.fr (C. Thinus-Blanc). 


\section{Introduction}

The rationale of the present work rests on two lines of studies: (a) developmental investigations of spatial abilities in children and (b) a paradigm used in comparative psychology and notably in studies with rats, which aims at understanding spatial cognition within a comparative perspective, linking investigations on nonhuman primates (Gouteux, Thinus-Blanc, \& Vauclair, 2001; Gouteux, Vauclair, \& Thinus-Blanc, 1999; Miller, Gouteux, Delpolyi, Santos, $\&$ Hauser, in preparation) with those carried out with young children (Gouteux \& Spelke, 2001).

The basic procedure that we use in our experiments is borrowed from a test initially proposed by Cheng (1986) with rats to address the question of the encoding of two types of environmental features, geometric configuration and local cues in an object retrieval task. Cheng demonstrated that, after being disorientated, rats only relied on geometric features (i.e., the shape of a rectangular box) instead of local cues to find a baited place (see also Gallistel, 1990).

Hermer and Spelke (1994, 1996) and Wang, Hermer, and Spelke (1999) have examined the same question in a series of studies with human children and adults. Children (mean age: 22 months) saw a desired toy being hidden in one of the corners of a rectangular homogeneous experimental chamber. After disorientation, the participants were asked to retrieve the toy. In one of the experiments, the chamber contained no distinctive landmark. In another one, a nongeometric feature that broke the symmetry of the experimental apparatus (a blue wall or a distinctive landmark placed in one corner of the chamber) was added. The authors consistently found that, when no information other than the shape of the environment was available, children searched equally often in the correct and in the rotationally equivalent corner and more frequently in these two than in the other two remaining corners. When a nongeometric information was added, children still divided their searches between the two rotationally equivalent corners and seemed to ignore the added salient cues. Unlike children, adults were able to use both geometric and the other available cues to guide their search.

Extension of this procedure with nonhuman primates (i.e., rhesus monkeys and tamarins) led us to show (Gouteux et al., 2001; Miller et al., in preparation) that monkeys, like rats, human adults, and children, relied on the information provided by the large-scale geometry of the room to retrieve a food reward when no other cue was available. However, contrary to 22-month-old children, rhesus monkeys and tamarins were also able to use (after disorientation) nongeometric information (a colored wall) to appropriately reorient in the experimental enclosure.

Turning now to developmental studies of spatial cognition, investigators have shown during infancy a pattern of change with age from egocentric to allocentric coding that is a consideration of the relative positions of objects independent of the infant's position. Such allocentric coding is apparent by 
16 months of age (Acredolo, 1978; Acredolo \& Evans, 1980). Other evidence of objective coding of space (i.e., in terms of distance information) has also been demonstrated in groups of children of similar age (e.g., Huttenlocher, Newcombe, \& Sandberg, 1994). The organization through ontogeny of travels in space based on the use of external cues has also been tested in an adaptation of the radial maze (Olton, 1978). Typically, 2-year-olds failed on an eight-arm radial maze, while 4-year-olds had significantly higher performance (Foreman, Arber, \& Savage, 1984). However, accuracy in spatial memory (an index of organized search strategies) continues to improve through childhood (e.g., Aadland, Beatty, \& Maki, 1985; Overman, Pate, Moore, \& Peuster, 1996). Increasing cognitive mapping skills with age have been demonstrated in other types of tasks such as object finding tasks (Cornell \& Heth, 1983) or navigation tasks requiring children to move efficiently between several locations in a large scale (e.g., Hazen, Lockman, \& Pick, 1978; Morrongiello, Timmey, Humphrey, Anderson, \& Skory, 1995).

However, only few investigations have studied how young children would code geometric (e.g., by using a representation of the shape of the environment) and nongeometric information within a given space. Although the work of Hermer and Spelke (1994, 1996) and Spelke and Hermer (1996) has clearly indicated the predominance of geometric information for reorientation in children and adults, this work also showed that young children (up to 3 years of age) did not use nongeometric cues after disorientation to locate an object in a rectangular space, albeit these cues were made very salient to them.

Our experiments seek to address this question in a novel way. In effect, instead of testing children after disorientation in a geometric real space, young participants are shown a model of the rectangular space in which a reward object is hidden. In the categorization of spatial tasks proposed by Weatherford (1985), our task corresponds to a model/small scale, namely a space that can only be observed or manipulated, while the task used for example by Hermer and Spelke (1994) can be categorized as a navigable/small scale that is a space that is large enough to permit travel in it. In our task, the child watches the experimenter hiding an attractive object in a box in one corner and then the rectangular space is rotated (the participant is requested to close his/her eyes for the duration of the rotation of the display). Afterwards, the child is invited to retrieve the reward.

This design presents some obvious differences with the original task studied by Cheng (1986) and by Hermer and Spelke (1994). In effect, (a) the rectangular box that we used is a sort of model of a real space, (b) the apparatus and not the participant is rotated in our experiments, and (c) because visual cues outside the box are available throughout testing, it is thus possible to evaluate the role of these cues in finding the correct corner. Notwithstanding these differences, the task at hand can tap the relative involvement of the same kind of spatial information (geometric and nongeometric) than that used in navigational tasks. 


\section{Experiment 1}

This experiment was designed to test the ability of 3-, 4-, and 5-year-old children to retrieve a previously hidden reward by only relying on the shape of a small rectangular apparatus.

\subsection{Participants}

Participants were divided into four age groups: Groups "3-year-old," "4-year-old," "5-year-old," and "adult." The "3-year-old" group comprised five boys and five girls ranging in age from 2.9 to 3.6 years (mean age: 3.2 years). The "4-year-old" group was composed of five boys and five girls ranging in age from 3.6 to 4.3 years (mean age: 3.9 years). The "5-year-old" group was made of five boys and five girls ranging in age from 4.5 to 5.8 years (mean age: 5.3 years) and the "adult" group comprised five men and five women ranging from 23 to 30 years of age (mean age: 27.4 years). All children were born from full-term pregnancies and suffered from no known health problems. They all attended a local preschool. Testing with parental consent (for the children) took place in one room of their preschool. Adults were tested in an experimental room in the lab.

\subsection{Apparatus}

The experimental apparatus $(80 \times 20 \times 15 \mathrm{~cm})$ was made of wood panels painted white. This apparatus contained no salient landmark and was symmetrical in its geometry to within $180^{\circ}$. Four opaque identical boxes (diameter $3 \mathrm{~cm}$ ) stood in the four corners of the experimental apparatus. Each box could contain a food reward (candy). Participants could not locate it unless they pulled up the box. The experimental apparatus was placed on the center of a table $(100 \times 100 \mathrm{~cm})$ where the participant, once seated, could see the entire apparatus (see Fig. 1). The table was located in the center of a room in which there were several furniture (for example, a bookcase, a plant, or wall pictures). Because the experiments were run in two different schools, the experimental rooms and environments were not identical for all participants. However, the mean surface of these rooms was comparable (i.e., about $15 \mathrm{~m}^{2}$ ) and contained approximately the same features.

\subsection{Procedure}

\subsubsection{Procedure for children}

The experimenter brought the child in front of the experimental apparatus and encouraged him/her to freely explore the apparatus for approximately $5 \mathrm{~min}$. The experimenter invited the child to look into each one of the four corner boxes. Then, the experimenter informed the participant that the participant would put a candy in one of the corner boxes (randomly chosen). He then explained that the apparatus would be rotated out of sight of the participant who would later be requested to 


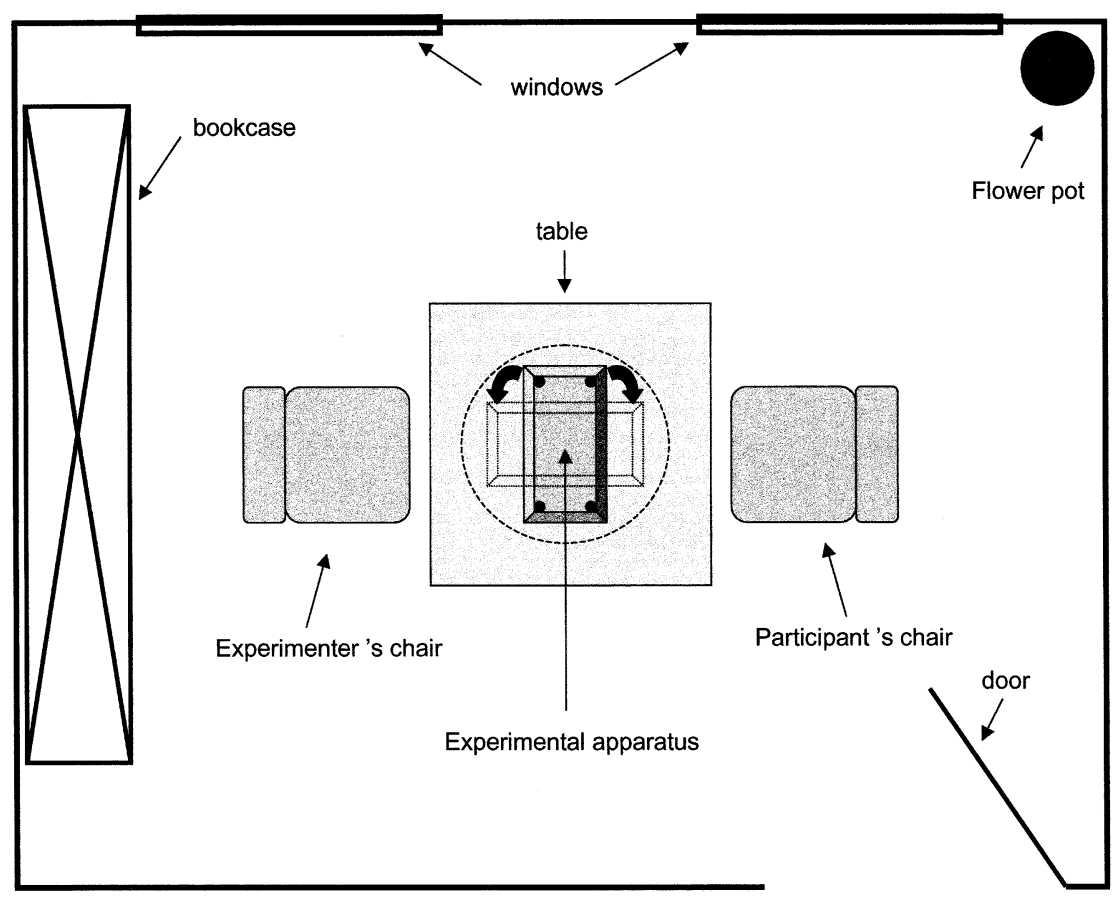

Fig. 1. Overhead view of the experimental environment. The rectangular box in the center of the drawing represents the small-scale model of the rectangular testing apparatus.

retrieve the hidden candy at the first trial. Before the disorientation occurred, the experimenter asked the child to point to the rewarded box. When the participant had clearly indicated the correct box, he or she was asked to close the eyes with the hands covering them (blind trials). Then, the experimental apparatus was rotated by the experimenter from exactly $90^{\circ}$ (clockwise or counterclockwise). The sequences of the rotations were pseudorandomly determined in order that each participant had no more than two consecutive rotations in the same direction and that the total number of clockwise or counterclockwise rotations was identical. When the experimental apparatus was left in the predetermined position, the participant was instructed to open his/her eyes and to search for the candy. The child was allowed to keep searching until $\mathrm{s} / \mathrm{he}$ found the reward and $\mathrm{s} / \mathrm{he}$ was again encouraged to retrieve it within the first choice. Then, the experimenter took another candy and asked the child to hide it in a new location within the box in order to begin a new trial.

\subsubsection{Procedure for adults}

The procedure used with the adults was similar to that used with the children except for the following. Adult participants were all university students recruited through announcements on the campus. They were offered 
credit for their participation to the experiment. The target used for the finding task was a plastic coin.

\subsubsection{Design}

Each participant was given a control search trial (eyes open during the rotation of the experimental apparatus) and six test trials (eyes closed during the rotation of the experimental apparatus). Each test trial consisted of one rotation trial in which the reward was hidden into one of four corner boxes of the experimental apparatus. During the experiment, each of the four boxes was used to hide the reward at least once and the order of the control/test trials was randomly chosen. The facing orientation of the experimental apparatus during all the experiment varied from trial to trial and was randomly determined with the restriction that approximately equal numbers of trials ended with participants facing with equal frequency the length and the width of the experimental apparatus.

\subsubsection{Coding and data analysis}

A search response was coded by the experimenter when the participant actually touched one of the boxes until the reward was found. The main analysis focused on the location on the participant's first contact with a box on each search trial but search behavior was recorded until the participant found the reward. Search location was coded along two dimensions in Experiments 1: it was coded as "geometrically appropriate" if the participant searched either at the correct corner that contains the reward (noted $\mathrm{C}$ ) or at its rotational equivalent (noted R) located on the same diagonal at $180^{\circ}$ and as "geometrically inappropriate" if otherwise ("near" corner noted as N and far "corner" noted as F). For each participant's first search trial, search at the geometrically appropriate vs. inappropriate corner was analyzed with a binomial test. Furthermore, paired-sample $t$ tests were run to determine whether searches at specific corners exceeded searches to other specific corners. Sample $t$ tests were run to analyze if searches at specific corners exceeded chance level. Finally, we tested if any improvement in performance was observed in the different age groups (independent-samples $t$ tests) for the geometrically correct corners.

\subsection{Results}

Fig. 2 presents the mean frequency of searches for each of the different corners and for each of the four groups of participants ("3-year-old," "4-year-old," "5-year-old," and "adult").

\subsection{1. "3-year-old" group}

The search patterns for the geometrically appropriate and inappropriate boxes are not different [paired-sample $t$ test, $t(9)=0.514, P>.05$ ], showing that the 3 -year-olds' searches were conducted at random. Search patterns also are random if one considers only the first test trial for individual participants. Thus, 4 of the 


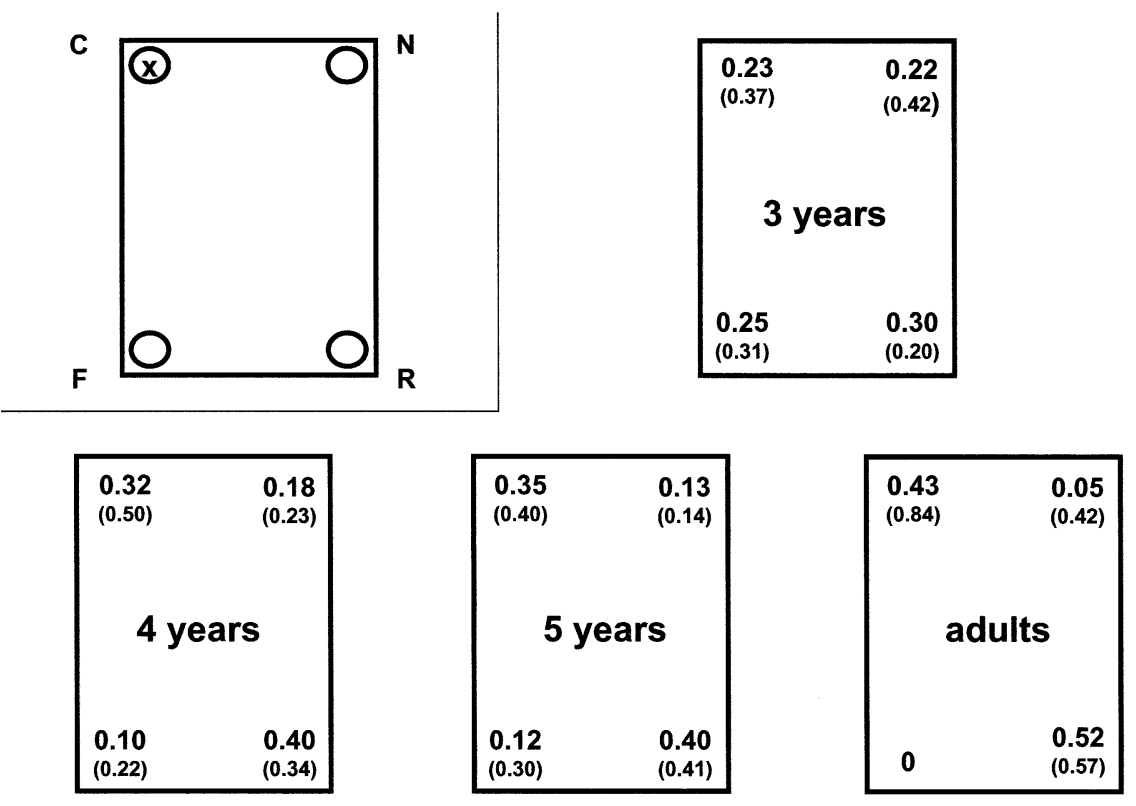

Fig. 2. Mean frequency of responses (and standard deviation) for all the participants over all the test trials of Experiment 1 for the "geometrically appropriate corners," i.e., the correct corner (noted C), the rotational equivalent corner (noted R), and the "geometrically inappropriate corners," i.e., the corner near the correct one (noted $\mathrm{N}$ ) and far the correct corner (noted F).

10 children searched the geometrically correct containers $(P>.20$, binomial test, ns). Children also searched in the correct corner and in its diagonal opposite with equal frequency during all test trials [paired-sample $t$ test, $t(9)=-0.885$, $P>.05]$ as well as on the first trial $(P>.30$, binomial test, ns). During the control trials, children searched in the correct container significantly more than expected by chance [one-sample $t$ test, $t(9)=2.946, P<.05$ ], indicating that they remembered where the target was hidden and were able to keep track of the location as the rectangular box was rotated.

\subsection{2. "4-year-old" group}

Participants in this group searched the geometrically appropriate boxes more than inappropriate ones, both on the first trial (binomial test, $P<.05$ ) and over the whole trial series $[t(9)=3.88, P<.005]$. Comparison of searches between the two geometrically appropriate corners reveals that participants showed no tendency to search more in the correct corner or the opposite corner, either on the first trial (binomial test, $P>.20$, ns) or on all trials $[t(9)=-0.889, P>.05]$. All participants also searched correctly on the control trials $[t(9)=6.5, P<.001]$. Searches in the geometrically correct diagonal for the "4-year-old" group reliably exceeded search in the same diagonal for "3-year-old" group $[t(18)=-2.191, P<.05]$. 


\subsection{3. "5-year-old" group}

As for the previous group of children, these participants tended to search geometrically appropriate boxes more than the geometrically inappropriate ones $[t(9)=4.39, P<.005]$. This effect is also apparent at the first trial $(P=.001$, binomial test). Children also searched in the correct box and in its diagonal opposite with equal frequency during all test trials $[t(9)=-0.38, P>.05]$ as well as on the first trial $(P>.20$, binomial test, ns). Furthermore, all participants searched correctly on the control trials (100\% of correct search). Finally, searches of this children's group in the geometrically correct diagonal reliably exceeded searches in the same diagonal compared with those of the "3-year-old" group $[t(18)=-2.537, P<.05]$. However, in this comparison, the performance of the children in this group did not differ from those of the "4-year-old" group $[t(18)=-0.418, P>.05]$.

\subsection{4. "Adult" group}

As for the older children, adult participants tended to search geometrically appropriate boxes more frequently than inappropriate boxes both on the first trial $(P=.001$, binomial test $)$ and over all test trials $[t(9)=17.67, P<.001]$. Comparison of searches between the two geometrically appropriate corners reveals that these participants showed no tendency to search the correct corner more than the rotational corner neither on the first trial $(P>.20$, binomial test, ns) or on all trials $[t(9)=-1.168, P>.05]$. Finally, all participants correctly searched on the control trials $(100 \%$ of correct searches). Searches made by this age group in the geometrically correct diagonal reliably exceeded searches in the same diagonal compared with the "3-year-old" group $[t(18)=-5.659, P<.001]$, the "4-year-old" group $[t(18)=-3.802, P<.001]$, and the "5-year-old" group $[t(18)=-3.207, P<.005]$.

\subsection{Discussion}

The scope of this experiment was to determine if children of different ages (3-, 4-, and 5-year-olds and adults) were able to find a hidden target by relying on the geometric information of a small-scale rectangular space. Unlike previous experiments (see, for example, Hermer \& Spelke, 1994, 1996), the children are not actively disoriented before searching. However, the only available information for the participant to retrieve the target are the geometric properties of the rectangular apparatus.

Our results show that 3-year-old children did not use the geometric information provided by the rectangular apparatus. Their search patterns were conducted at random. In contrast, the two other groups of children (3-and 4-year-olds) searched reliably more often in the geometrically correct diagonal (the one that contains the correct and the geometrically correct corners) than in the geometrically incorrect corners. These data clearly indicate that children older than 3 years of age are able to use the geometrical properties in a small-scale rectangular model to "reorient." 
The search performances of the adults were optimal and in all cases better than the children scores.

These results will be discussed with results obtained in similar situations, whereby participants could move around (see General discussion), but it appears that the encoding and use of geometrical information is closely related to the scale of the environmental apparatus. Different mechanisms could be at work while processing different spaces (locomotor vs. manipulatory). To test for this hypothesis, it would be of interest to examine the development of children's search abilities when more salient information (a colored cue) is provided to the shape of the rectangular small-scale model. The next experiment was designed to test this issue.

\section{Experiment 2}

In Experiment 2, four new groups of participants were given the same target search task used in Experiment 1. However, a nongeometric landmark was added in the rectangular environment.

\subsection{Participants}

Four new age groups of participants were tested: the "3-year-old" group was made of five boys and five girls (mean age: 3.1 years); the "4-year-old" group was composed of five boys and five girls (mean age: 4.08 years); the "5-year-old" group was made of five boys and five girls (mean age: 5.1 years); and the "adult" group was composed of five men and five women (mean age: 26.8 years).

\subsection{Apparatus}

The same rectangular apparatus as in Experiment 1 was used. A rectangular plastic yellow cue $(20 \times 15 \mathrm{~cm})$ was added on one of the small walls of the apparatus so as to cover it completely and make this wall fully colored in yellow.

\subsection{Procedure}

\subsubsection{Procedure for children and adults}

The procedure was the same as in the previous experiment, except that each participant was asked to point out the colored wall of the apparatus and also to tell what was its color. Each participant was tested on six test trials and on one control trials where the rectangular apparatus could be rotated from exactly $180^{\circ}$ or $90^{\circ}$ (clockwise and counterclockwise). The sequences of rotations were pseudorandomly determined in order that participants had no more than two consecutive rotations in the same direction and of the same degree of rotation and also that the total number of clockwise or counterclockwise rotations was 
identical. Furthermore, the sex of the participant, the box rewarded, the trial order (control vs. test), and the degree of rotation of the experimental apparatus were orthogonally counterbalanced across participants.

\subsubsection{Design}

The same experimental design as in Experiment 1 was used here.

\subsubsection{Coding data and analysis}

The main analysis was the same as in the previous experiment (i.e., "geometrically appropriate" and "geometrically inappropriate"). However, a new coding dimension was added: a search was coded as "adjacent" to the landmark if the participant searched either at the correct corner (noted C) or at the corner nearest to C (corner N) and as "opposed" to the landmark if otherwise (corners R and F). Finally, a binomial test was run on each participant's first search trial for the adjacent vs. opposed corner.

\subsection{Results}

Fig. 3 presents the mean frequency of searches for each of the different corners as a function of the four groups of participants ("3-year-old," "4-year-old," "5-year-old," and "adult").

\subsection{1. "3-year-old" group}

The search patterns for the geometrically appropriate and inappropriate boxes did not differ from chance $[t(9)=0.264, P>.05]$. If one considers only the first test trial, search patterns were random $(P>.20$, binomial test, ns). Considering the adjacent corners to the landmark (noted $\mathrm{C}$ and $\mathrm{N}$ ) and the opposed corners (noted $\mathrm{R}$ and $\mathrm{F}$ ), search patterns did not differ from chance neither on the first trial $(P>.30$, binomial test, ns) nor on all trials $[t(9)=-1.55, P>.05]$. Also, comparison of the searches at the two landmark's corners (noted $\mathrm{C}$ and $\mathrm{N}$ ) showed no differences on the first trial $(P>.20$, binomial test, ns) or on all trials $[t(9)=-0.612, P>.05]$. During control trials, children searched in the correct container significantly more than expected by chance $[t(9)=4.125, P<.005]$. This result indicates that they were able to keep track of the location of the correct box and that they remembered where the target was hidden as the rectangular box was rotated.

\subsection{2. "4-year-old" group}

Participants in this group tended to search to the geometrically appropriate and inappropriate boxes with equal frequencies, both on the first trial $(P>.20$, binomial test, ns) and over the trial series $[t(9)=0.487, P>.05]$. However, searches at the two boxes near the landmark (correct box and incorrect near box) were more frequent than searches at the two distant boxes, geometrically correct and incorrect far box $[t(9)=3.28, P<.001]$. Comparison of the searches 


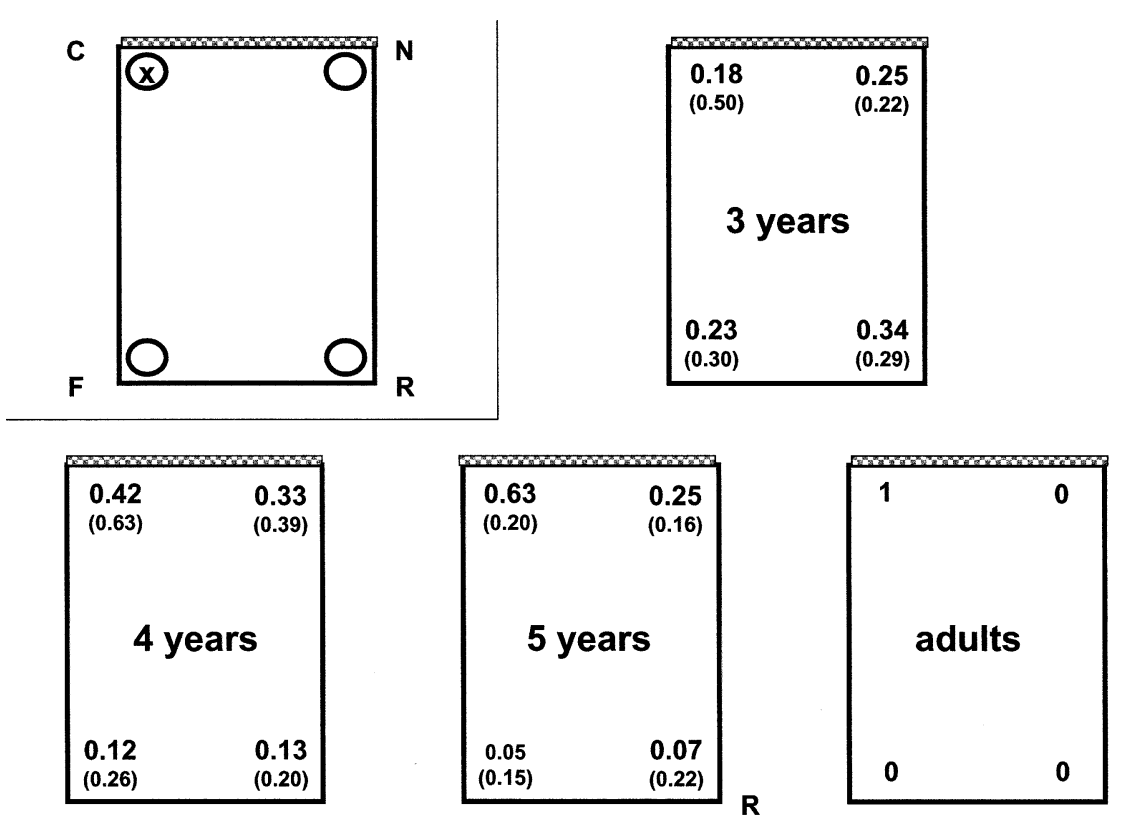

Fig. 3. Mean frequency of responses (and standard deviation) for all the participants over all the test trials of Experiment 2 for the correct corner (noted C), the rotational equivalent corner (noted R), the near corner (near the correct corner, noted N), and the far corner (far from the correct corner, noted F).

at the two landmark's corners showed no tendency to search the correct corner more than the incorrect near corner on the first trial $(P>.20$, binomial test, ns) or on all trials $[t(9)=0.493, P>.05]$. All participants also searched correctly on the control trials $[t(9)=2.946, P<.05]$. In this experiment, the "4-year-old" children searched reliably more accurately than the "3-year-old" group (Experiment 2 ) in the adjacent corners [C and $\mathrm{N} ; t(18)=2.92, P<.01]$.

\subsection{3. "5-year-old" group}

Searches to the geometrically appropriate corners exceeded searches to the inappropriate corners over the trial series $[t(9)=6, P<.001]$ and on their first trials $(P<.01$, binomial test). Furthermore, searches at the two boxes near the landmark (correct box and incorrect near box) were more frequent than searches at the two distant boxes [geometrically correct and incorrect far box; $t(9)=12.65, P<.001]$. Comparison of searches at the two landmark's corners reveals that search to the correct corner were more frequent than at the incorrect near corner on the first trial $(P<.05$, binomial test $)$ or on all trials $[t(9)=6.273$, $P<.001]$. All participants searched correctly on the oriented trial $[t(9)=6.5$, $P<.001]$. Finally, in Experiment 2, searches to the adjacent corners were better in the "5-year-old" group than in the "3-year-old" group $[t(18)=6.495$, $P<.001]$ and in the "4-year-old" group $[t(18)=3.113, P<.01]$. 


\subsection{4. "Adult" group}

Searches at the geometrically incorrect corners at the opposed corners or at the near corner never occurred for the adult participants either on the first or on all the trials. Thus, no statistical analysis could be done (each S.D. $=0$ ). All participants searched in the correct corner in each one of the experimental tests or control trials ( $100 \%$ of correct searches). Finally, participants' searches to the adjacent corners were better (Experiment 2) in the "adult" group compared to the "3-year-old" group $[t(18)=7.964, P<.001]$ and to the "4-year-old" group $[t(18)=4.294$, $P<.001]$ but not between the "adults" group and the "5-year-old" group $[t(18)=1.405, P>.05]$.

\section{General discussion}

The aim of the present experiments was to investigate in children of different ages the use of geometric configuration and local cues in an object retrieval task. Testing was also conducted with a group of adults. Unlike previous experiments that took place in a real-space and involved navigation, we used a small-scale model of a rectangular space, which could be only observed or manipulated. In the first experiment, the geometry of the box was the sole information allowing the participants to make a distinction between, on the one hand, the two geometrically correct corners and, on the other hand, the two remaining corners. In the second experiment, a visual cue affixed to one of the walls permitted to differentiate the correct corner from its rotationally equivalent one and a fortiori from the other two corners. In both experiments, participants had to keep their eyes closed while the whole apparatus was rotated excepted for one control trial where they could watch while the tray was being rotated.

The data of Experiment 1 show that 3-year-old children did not make use of the geometry of the apparatus but searched equally in the four corners. In contrast, 4- and 5-year-old children concentrated their search to the geometrically correct corners, although they did not reach adult's performance levels. The presence of a conspicuous visual cue close to the correct corner in Experiment 2 had no effect on the performance of the 3-year-old children. In contrast, the "4-year-old" group searched more frequently in the correct corner than in the geometrically correct one, but most of their errors bear on the geometrically incorrect corner close to the cue. At 5 years of age, children predominantly choose the correct corner, and they did so in the same proportion as adults. However, 5-year-old children's errors were still concentrated at the corner close to the visual cue. In both experiments, children from all age groups and adults correctly performed during control trials (with the eyes opened).

In comparison with the results obtained in similar situations whereby participants could move around (e.g., Hermer \& Spelke, 1994), Experiment 1 yields a rather different pattern of data. In effect, it appears that the coding of environmental geometric features develops later in a small-scale setup than in a 
navigable setup, namely within locomotor space. Thus, toddlers of 24 months of age tested by Hermer and Spelke (1994) were already capable of distinguishing the geometrically correct corners from the other ones. In the present experiment, this ability emerges only at 4 years of age, and even at 5 years, children do not perform as accurately as adults do.

Such results suggest that different types of spatial processing might be involved according to the kind of task participants are faced with (see also, Weatherford, 1985). In a room where participants can move about, locomotor activity (e.g., in terms of translations or rotations) generates ever-changing perspective points and visual scenes. Spatial coding, regardless of the type of information taken into account, implies a continuous updating of the participant's successive positions within the environment that constitutes a stable reference frame. In contrast, when the participant is still, even if eye, head, and trunk movements provide changes of perspective points (though to a lesser extent than locomotor activity), the body itself constitutes a stable reference by comparison with the experimental layout that is rotated by the experimenter. In this latter case, the participant is passive in relation with the rotation that he/she has not the opportunity to watch (the eyes being closed) while the rotation is completed.

As a general point, we could consider that a small-scale space and a navigational space differ with respect to the action programs they entail. Thus, a small-scale space, i.e., space of manual grasping, is entirely open to immediate and visual apprehension, whereas an individual progressively discovers navigational space through his/her displacements. The consequence of such a difference in capturing these two spaces is that manual space stresses the need of an action program centered on the goal such as hit and grasp the rewarded object, where navigational space relies more on planing the displacements by considering the global layout of the environment.

Indeed, several studies have demonstrated that getting actively acquainted with a situation induces better performance levels than being passively shown the experimental layout (Peruch, Vercher, \& Gauthier, 1995; Tong, Marlin, \& Frost, 1995). As a matter of fact, the coding of geometric features would appear earlier when children can actively move within the experimental situation. A review of the role of active locomotor movements in spatial cognition has been performed by Cohen and Cohen (1985) with an emphasis on development. From a literature, which is somewhat controversial, these authors pinpoint the fact that active movement mostly facilitates the acquisition of spatial knowledge for large-scale environments but "it presents little advantage when the space can be viewed in its entirety from single vantage points " (Cohen \& Cohen, 1985, p. 217). Given that the testing environment used by Hermer and Spelke was small $(6 \times 4 \mathrm{ft})$ and that locomotion of the participants was very limited in this environment, it is unlikely that the factor of active vs. passive locomotion can explain the delay we observe in our tasks compared to the navigational search tasks such as those used by Hermer and Spelke. 
Presson and Hazelrigg (1984) have proposed another distinction that also seems relevant to account for the developmental delay in the appearance of the processing for coding geometric properties in a small-scale task. These authors oppose "primary" to "secondary" spatial representations. Primary spatial representations are constructed on the basis of the organism's experience within an actual environment. That is the case for animals and young children when they move and orient in their respective usual space. In contrast, secondary spatial representations result from consulting a symbolic representation of a situation such as reading a map or observing a small-scale model of a real environment. In such a case, the spatial configuration is an external object (i.e., separate from the participant) instead of being a medium where the organism is immersed. Consequently, the orientation of the configuration when it is presented affects the way it is subsequently used. For instance, Presson and Hazelrigg have demonstrated that the use of secondary spatial representations in transfer tests is difficult from a different orientation in the actual environment (because of contralignment or misalignment effects). In contrast, the use of primary spatial representations that ipso facto are not initially oriented is independent from any perspective point. This distinction seems likely to account for the data of Experiment 1 of the present study (for similar difficulties to use a model of the environment in young children, see Deloache,1987).

With a small-scale situation, the orientation of the layout by reference to the child's position and its rotation may have interfered with the coding of geometric features in the 3-year-old group. For this particular task, 3-year-old children may have used an egocentric frame of reference. In doing so, they must have encoded and remembered the location of the rewarded box as being either far or close from them and on the right or on the left of their own location. This interpretation could explain why 3-year-old children were unable to correctly find the target after the rotation of the tray. In effect, the observed pattern of responses was randomly dispersed all through the four corners of the box. Such a pattern could indicate that they were searching without having taken into account the rotation of the tray. By contrast, the oldest children and the adults could have used an allocentric frame of reference and thus could have taken into consideration the displacement of the tray during the test. The use of such coding might explain why their performance were better than those of the younger children.

In Experiment 2, where a conspicuous cue allowed participants to locate the correct corner, 3-year-old children did not make any use of this information and behaved the same way as the 2-year-old toddlers in Hermer and Spelke's (1996) study. In marked contrast, 4- and 5-year-old children were able to rely on the nongeometrical cue to help them finding the reward. A peculiar feature of most of the 4-year-olds, and of a minority of the 5-year-olds, concerns the nature of the errors. Most of their errors were concentrated at the geometrically incorrect corner close to the visual landmark. This point deserves attention because it reveals that besides geometric and discrete local cues, children make 
also use of a topological property, i.e., vicinity. Indeed, adequately locating the correct corner implies the use of both geometric and local information. Vicinity errors could mean that the visual cue has been used alone. Such a behavior is at odds with other data in children and animals showing a predominance of geometric coding (e.g., Cheng, 1986; Kamil \& Jones, 1997; Margules \& Gallistel, 1988; Wang et al., 1999) and also at odds with the behavior of adults and nonhuman primates who make a joint use of geometric and local information (Gouteux et al., 2001; Hermer \& Spelke, 1994, 1996; HermerVasquez, Spelke, \& Katsnelson, 1999). Such behaviors may reflect a beginning of a combination of the geometric coding system with local (correct responses) and topological (vicinity errors) information.

However, another hypothesis concerning the predominant use of the nongeometric cue by most 4-year-old and also some 5-year-old children can be expressed. According to Spelke and Hermer (1996), the ability to use nongeometric information does not emerge in an all-or-none fashion. Briefly, children appear to use the nongeometrical landmark in a more flexible way with increasing age. Despite the fact that our experimental environment was different form the one used by Hermer and Spelke (1994), we can hypothesize that even in a manipulatory space the use of landmarks emerges progressively in children. Before 4 years of age, children do not take into account this type of information. Gradually, after that age, cognitive development of the children enables them to use the nongeometrical information from a dominant use at 4 years of age to a more flexible way at 5 years of age when they are capable to conjointly take into account geometric and nongeometric information and to perform as well as adults.

The source of that flexibility seems tightly related to the development of language and more precisely to spatial language. In effect, according to Hermer-Vasquez et al. (1999) and Spelke and Hermer (1996), language that $2-3$-year-old children begin to produce and use correctly at about 6 years of age appears to provide an especially useful medium for representing conjunctions of spatial and nonspatial properties of the environment. These ages match the one when children begin to use imperfectly nongeometrical information (at about 2.5 years) and the one where they use these spatial information in an optimal way (at about 5.5 years) in our experiments and also in previous navigational experiments (Hermer-Vasquez et al., 1999; Spelke \& Hermer, 1996). Taken together, our findings strengthen the idea that language is necessary to human beings for combining these two different aspects of core knowledge (shape and landmark information). This specific human ability seems to be at work in different types of environments (locomotor and manipulatory) and could underline a common mechanism devoted to general orientation. This mechanism could have emerged across evolution and may account for the better flexibility of the human being with respect to other animals. However, the nature and functioning of this possible mechanism require further investigation. 


\section{Acknowledgments}

This research was funded by the French Ministry of Research (Grant 97-5003224 to S.G.) and by the National Center of Scientific Research (CNRS, Marseille, France). We thank E.S. Spelke for useful discussions on this work and J. Kroi for helping in the collection of data. Part of this work was previously presented at the Seventh Annual Meeting of the Cognitive Neuroscience Society (April 2000, San Francisco, USA).

\section{References}

Aadland, J., Beatty, W. D., \& Maki, R. H. (1985). Spatial memory in children and adults assessed in the radial maze. Developmental Psychobiology, 18, 163-172.

Acredolo, L. P. (1978). Development of spatial orientation in infancy. Child Development, 14, $224-234$.

Acredolo, L. P., \& Evans, D. (1980). Developmental changes in the effects of landmarks on infant spatial behavior. Developmental Psychology, 16, 312-318.

Cheng, K. (1986). A purely geometric module in the rat's spatial representation. Cognition, 23, $149-178$.

Cohen, S. L., \& Cohen, R. (1985). The role of activity in spatial cognition. In: R. Cohen (Ed.), The development of spatial cognition (pp. 199-221). Hillsdale, NJ: Lawrence Erlbaum Associates.

Cornell, E. H., \& Heth, C. D. (1983). Spatial cognition: gathering strategies used by preschool children. Journal of Experimental Child Psychology, 35, 93-110.

Deloache, J. S. (1987). Rapid change in the symbolic functioning of very young children. Science, $238,1556-1557$.

Foreman, N., Arber, M., \& Savage, J. (1984). Spatial memory in preschool children. Developmental Psychobiology, 17, 129-137.

Gallistel, C. R. (1990). The organization of learning. Cambridge, MA: MIT Press.

Gouteux, S., \& Spelke, E. (2001). Children's use of geometry and landmarks to reorient in an open space. Cognition, 81, 119-148.

Gouteux, S., Thinus-Blanc, C., \& Vauclair, J. (2001). Rhesus monkeys use geometric and non geometric information during a reorientation task. Journal of Experimental Psychology: General, 130, $505-519$.

Gouteux, S., Vauclair, J., \& Thinus-Blanc, C. (1999). Reaction to spatial novelty and exploratory strategies in baboons. Animal Learning and Behavior, 27, 323-332.

Hazen, N. L., Lockman, J. J., \& Pick, H. L. (1978). The development of children's representations of large-scale environments. Child Development, 49, 623-636.

Hermer, L., \& Spelke, E. (1994). A geometric process for spatial reorientation in young children. Nature, 370, 57-59.

Hermer, L., \& Spelke, E. (1996). Modularity and development: the case of spatial reorientation. Cognition, 61, 195-232.

Hermer-Vasquez, L., Spelke, E., \& Katsnelson, A. (1999). Source of flexibility in human cognition: dual-task studies of space and language. Cognitive Psychology, 39 (1), 3-36.

Huttenlocher, J., Newcombe, N., \& Sandberg, E. H. (1994). The coding of spatial location in young children. Cognitive Psychology, 27, 115-147.

Kamil, A. C., \& Jones, J. E. (1997). The seed-storing Corvid Clark's nutcracker learns geometric relationships among landmarks. Nature, 390, 276-279.

Margules, J., \& Gallistel, C. R. (1988). Heading in the rat: determination by environmental shape. Animal Learning and Behavior, 16, 404-410. 
Miller, C., Gouteux S., Delpolyi A., Santos L., \& Hauser M. (in preparation). Primate spatial representations: coordinated use of geometric and landmark information by cotton top tamarins.

Morrongiello, B. A., Timney, B., Humphrey, K., Anderson, S., \& Skory, C. (1995). Spatial knowledge in blind and sighted children. Journal of Experimental Child Psychology, 59, 211-233.

Olton, D. S. (1978). Characteristics of spatial memory. In: S. H. Hulse, H. Fowler, \& W. K. Honing (Eds.), Cognitive processes in animal behavior (pp. 341-373). Hillsdale, NJ: Lawrence Erlbaum Associates.

Overman, W. H., Pate, B. J., Moore, K., \& Peuster, A. (1996). Ontogeny of place learning in children as measured in the radial arm maze, Morris search task, and open field task. Behavioral Neuroscience, 10, 1205-1228.

Peruch, P., Vercher, J. L., \& Gauthier, G. M. (1995). Acquisition of spatial knowledge through visual exploration of simulated environments. Ecological Psychology, 7, 1-20.

Presson, C., \& Hazelrigg, M. (1984). Building spatial representations through primary and secondary learning. Journal of Experimental Psychology: Learning, Memory and Cognition, 10, 716-722.

Spelke, E., \& Hermer, L. (1996). Early cognitive development: objects and space. In: R. Gelman, $\&$ T. Kit-Fong $\mathrm{Au}$ (Eds.), Perceptual and cognitive development (pp. 71-114). New York: Academic Press.

Tong, F. H., Marlin, S. G., \& Frost, B. J. (1995). Visual-motor integration and spatial representation in a visual virtual environment. Investigative Ophthalmology and Visual Science, 36, 1679.

Wang, R. F., Hermer, L., \& Spelke, E. S. (1999). Mechanisms of reorientation and object localization by children: a comparison with rats. Behavioral Neuroscience, 113, 475-485.

Weatherford, D. L. (1985). Manipulating spatial information from different environments: models to neighborhoods. In: R. Cohen (Ed.), The development of spatial cognition (pp. 41-71). Hillsdale, NJ: Lawrence Erlbaum. 